\section{TICKER}

Krankenstand 2009: Beschäftigte fast einen Tag länger krankgeschrieben Der Krankenstand ist im letzten Jahr bundesweit sprunghaft angestiegen, wie die Techniker Krankenkasse (3,4 Mio. Versicherte) meldet. Die Arbeitnehmer waren durchschnittlich 12,1 Tage krankgeschrieben, fast einen Tag mehr als 2008. Neben einer erneuten Zunahme psychischer Erkrankungen $(+13,9 \%)$ ist der Anstieg von $7,7 \%$ vor allem auf zwei Erkältungswellen im Januar und November zurückzuführen. Im November wurde - aus Angst vor einer erhöhten Ansteckungsgefahr durch die Schweinegrippe - den Beschäftigten oft geraten, bei Erkältungssymptomen lieber zu Hause zu bleiben.

(KE) -

Mäßiger Alkoholkonsum für die schlanke Linie? Frauen, die regelmäßig, aber in

Maßen alkoholische Getränke zu sich nehmen, haben ein geringeres Risiko, übergewichtig zu werden als Abstinenzlerinnen. Dies ist das überraschende Ergebnis einer Studie mit rund 19220 Amerikanerinnen im Alter über 39 Jahren. Im Laufe von 13 Jahren nahm die gesamte Studienpopulation an Gewicht zu. Die geringste Zunahme wurde jedoch bei Frauen registriert, die täglich alkoholische Drinks in mäßigen Mengen (15-30 g) konsumierten. Sie hatten ein fast um $30 \%$ niedrigeres Risiko, zuzunehmen als Frauen, die keinerlei Alkohol tranken.

(мо) -

Archives of Internal Medicine 2010;170(5):453 DOI: 10.1001/archinternmed.2009.527

\title{
RENALE HYPERTONIE
}

\section{Cola und Co. treiben den Blutdruck hoch}

Schon zwei bis drei Gläser Cola, Limo oder süßer Fruchtsaft täglich treiben den Blutdruck hoch. Schuld daran ist der hohe Fruktosegehalt der Softdrinks. Kritisch wird es bereits ab $74 \mathrm{~g}$ Fruchtzucker pro Tag. Das sind etwa 2,5 Softdrinks pro Tag. Zu diesem Ergebnis kam eine Studie, in der 4528 Probanden ohne anamnestisch bekannten Bluthochdruck nach ihrem Konsum von Fruchtsäften, Softdrinks, Backwaren und Süßigkeiten befragt worden waren. Die täglich zugeführte Fruchtzuckermenge korrelierte eng mit dem Hypertonierisiko. Die klinische Relevanz: In den letzten Jahrzehnten hat der Fruktosegehalt in der Nahrung stetig zugenommen. Amerikaner konsumieren heute etwa $30 \%$ mehr Fruchtzucker als vor 20 Jahren. (Kw) 42. Jahrestagung der American Society of Nephrology (ASN) in San Diego/Kalifornien (USA).

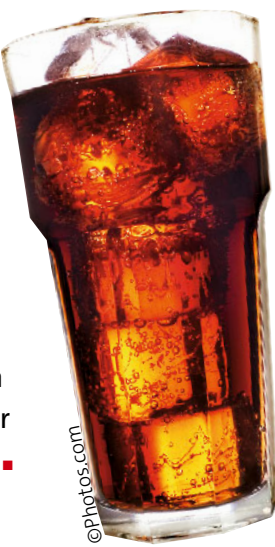

\section{NEUES ZENTRUM IN TÜBINGEN}

\section{Endlich mehr Hilfe für Patienten mit seltenen Erkrankungen}

Im Januar 2010 wurde an der Universität Tübingen das bundesweit erste Zentrum für Seltene Erkrankungen (ZSE-Tübingen) eröffnet. Patienten, die sich hierhin wenden, werden durch interdisziplinäre Spezialistenteams betreut und ihre Krankheiten weiter erforscht. Eine Erkrankung gilt in der EU dann als seltene Krankheit (Orphan Disease), wenn weniger als 5 pro 10000 Einwohnern betroffen sind. Man kennt 6000 - 8000 verschiedene Krankheitsbilder, 80\% davon haben genetische Ursachen. Die Pa- tienten finden meist jahrelang keine Hilfe. Erfreulicherweise engagiert sich mittlerweile auch die Pharmaindustrie zunehmend im Bereich der Orphan Drugs. (sт) .

Kontakt:

Universitätsklinikum Tübingen Zentrum für Seltene Erkrankungen (ZSE-Tübingen) Calwerstr. 7, D-72076 Tübingen, Tel.: 07071/29-85170 E-Mail: info@zse-tuebingen.de www.zse-tuebingen.de

\section{UROLOGEN DRÄNGEN AUF RASCHE UMSETZUNG}

\section{Neue S3-Leitlinie Prostatakrebs}

Die im Herbst 2009 veröffentlichte interdisziplinäre Leitlinie zum Prostatakarzinom soll so schnell wie möglich im Behandlungsalltag umgesetzt werden. Die Deutsche Gesellschaft für Urologie e.V. (DGU) und der Berufsverband der Urologen haben in der S3-Leitlinie das verfügbare Wissen zu Früherkennung, Diagnostik und Behandlung des Prostatakarzinoms zusammengetragen. Wichtigste Neuerungen: Die erste PSA-Untersuchung wird bereits für den 40-jährigen Mann empfohlen, die Patienten sollen besser über Diagnostik und Therapiemöglich- keiten aufgeklärt werden und ein „Active Surveillance" soll als gleichrangige Therapieoption für Patienten mit nicht metastasiertem Prostatakarzinom gelten.

(st) -
Die ärztliche Leitlinie finden Sie unter www.urologenportal.de, einen Patientenratgeber unter www.krebsgesellschaft.de/wub II_pat,120885.html.

In gedruckter Form ist der Ratgeber über die Deutsche Krebsgesellschaft erhältlich. 